\title{
Energy use efficiency in soybean crops in different regions of Brazil
}

\section{Eficiência energética na cultura da soja cultivada em diferentes regiões brasileiras}

\author{
Roni Fernandes Guareschi ${ }^{1 *}$; Marcio dos Reis Martins ${ }^{1}$; Leonardo Fernandes \\ Sarkis ${ }^{2}$; Bruno Jose Rodrigues Alves ${ }^{3}$; Claudia Pozzi Jantalia ${ }^{3}$; Robert Michael \\ Boddey $^{3}$; Segundo Urquiaga ${ }^{3}$
}

\section{Highlights:}

Energy efficiency analysis helps to identify economic and environmental bottlenecks.

Low soil fertility resulted in high use of energy associated with fertilizers.

Energy efficiency should be assessed in different representative regions.

\begin{abstract}
The soybean crop in Brazil has been growing in area and productivity in recent years and the analysis of its energy efficiency is very important to guarantee the sustainability of the production system. Assessment of energy efficiency (EE) enables the evaluation of the sustainability of agrosystems, as well as decision-making regarding the reduction in production costs and negative environmental impacts. In this context, the objective of this study was to assess energy efficiency of soybean in different regions of Brazil. For this purpose, 29 areas of soybean across the major producing states were assessed. Energy inputs and outputs of agricultural operations and/or agricultural inputs were calculated by multiplying the amount used by their calorific value or energy coefficient at each stage of production. Energy efficiency was calculated as the ratio between the total output energy and the total input energy during the production process. For every MJ of energy consumed in the production of soybean crop, 6.1; 6.7; 7.1 and 7.2 MJ of energy were produced in the form of grain, respectively in the areas assessed in the Midwest, northeast, southeast and south regions of Brazil. Generally, the main energy expenditure on soybean cultivation in different regions of Brazil was with fertilizers, seeds and herbicides. The adverse weather conditions of the year / harvest evaluated in the south-central region of Brazil resulted in low soybean yields and consequently resulted in lower energy efficiency in these regions. The evaluation of energy efficiency in soybean crops to be representative must be carried out in different regions and edaphoclimatic conditions.
\end{abstract}

Key words: Energy consumption. Glycine max. Grain yield.

1 Pós-Doutorando, Programa de Pós-Graduação em Ciência do Solo, Universidade Federal Rural do Rio de Janeiro, UFRRJ, Seropédica, RJ, Brasil. E-mail: guareschiecotarelli@hotmail.com; marcio.dos.reis.martins@gmail.com

2 Discente de Mestrado, Programa de Pós-graduação em Ciência do Solo, Universidade Federal de Lavras, UFLA, Lavras, MG, Brasil. E-mail: leonardo.sarkis@hotmail.com

3 Pesquisadores, Empresa Brasileira de Pesquisa Agropecuária, EMBRAPAAgrobiologia, CNPAB, Seropédica, RJ, Brasil. E-mail: bruno.alves@embrapa.br; claudia.jantalia@embrapa.br; robert.boddey@embrapa.br; segundo.urquiaga@embrapa.br

* Author for correspondence

Received: Apr. 15, 2020 - Approved: Aug, 18, 2020 


\section{Resumo}

A cultura da soja no Brasil vem crescendo em área e produtividade nos últimos anos e, com isso, a análise de sua eficiência energética é muito importante para garantir a sustentabilidade do sistema produtivo. A avaliação da eficiência energética (EE) pode evidenciar a sustentabilidade dos agrossistemas e a tomada de decisões relativas à redução dos custos de produção e poluição do ambiente. Diante deste contexto, o objetivo deste trabalho foi avaliar a eficiência energética na cultura da soja em diferentes regiões brasileiras. Para isso, foram avaliadas 29 áreas de soja distribuídas pelos principais estados produtores desta cultura. As entradas e saídas de energia das operações agrícolas e/ou insumos utilizados foram calculadas pela multiplicação da quantidade utilizada pelo seu poder calorífico ou coeficiente energético em cada etapa de produção. A eficiência energética foi obtida pela razão entre a quantidade de energia total de saída e o consumo total de energia durante o processo produtivo. Para cada MJ de energia consumida na produção da soja, 6,1; 6,7; 7,1 e 7,2 MJ de energia foram produzidos na forma de grãos de soja, respectivamente nas áreas avaliadas nas regiões Centro-Oeste, Nordeste, Sudeste e Sul do Brasil. Em uma média geral, os principais gastos energéticos no cultivo de soja em diferentes regiões do Brasil foram com fertilizantes, sementes e herbicidas. As condições climáticas adversas do ano/safra avaliado na região centro-sul do Brasil resultaram em baixas produtividades de soja e, consequentemente, interferiram na menor eficiência energética nessas regiões. A avaliação da eficiência energética na lavoura de soja para ser bem representativa deve ser realizada em diferentes regiões e condições edafoclimáticas.

Palavras-chave: Consumo energético. Glycine max. Produção de grãos.

\section{Introduction}

Considering the projection and/or estimation of world soybean production for the 2017-2018 crop year, Brazil stands out as the second largest producer of soybean (110 Mt), only behind the United States, with a production of $119 \mathrm{Mt}$ of soybean (United States Department of Agriculture [USDA], 2018). This grain production is associated with techniques highly dependent on energy consumption primarily from fossil fuel burning resulting in significant amounts of $\mathrm{CO}_{2}$ emission to the atmosphere.

The assessment of energy efficiency (EE) in agriculture can help to identify the energy bottlenecks of the adopted cropping systems, with the intention of finding energy-saving technologies (especially for fossil-fuel energy). The use of fuel, fertilizers, pesticides, irrigation, and the manufacture of machines and implements are examples of items that consume great amounts of energy in agriculture (Cunha et al., 2015; Chen et al., 2018). Therefore, studies on EE in agriculture contribute to the assessment of the sustainability of agricultural systems.
There are several ways to estimate the EE of a crop, such as the amount of energy used per unit mass of harvested product (Alluvione, Moretti, Sacco, \& Grignani, 2011) or the energy content of the crop per unit of energy used to produce it, in $\mathrm{J} \mathrm{J}^{-1}$ (Cunha et al., 2015). The literature presents a wide variation in the values of EE for soybean crops cultivated in Brazil. For example, several EE values have been reported for soybean crops: 5.47 (Melo et al., 2007); 18,64 (A. T. Campos, Klosowsk, Souza, Zanini, \& Prestes, 2009); 7.81 (Assenheimer, Campos, \& Gonçalves, 2009); 4.27 (Mourad \& Walter, 2011); 9.80 (Santos, Spera, Fontaneli, \& Dreon, 2011); 5.30 (Riquetti, 2014); and 13.66 (Ferreira, Neumann, \& Hoffmann, 2014). In most of these studies, energy was primarily attributed to fuels (A. T. Campos et al., 2009; Ferreira et al., 2014), fertilizers (Mourad \& Walter, 2011; Riquetti, 2014), herbicides (Assenheimer et al., 2009; Riquetti, 2014) and agricultural machinery (Melo et al., 2007; A. T. Campos et al., 2009).

These wide variations in the EE values of soybean crops in Brazil may be attributed to differences in 
crop productivity and crop management adopted in each locality. It is necessary to determine the EE of various representative localities to decrease these discrepancies and increase the representativeness of the EE results of soybean crops.

The detailed description of the practices and inputs that have an effect on EE in agriculture contributes to the establishment of the best practices for an environmentally and economically more efficient agricultural production. Thus, the objective of the present study was to assess EE in soybean crops in different regions of Brazil in the 2014-2015 crop year.

\section{Materials and Methods}

This was an exploratory study that followed the methodological approach used in multiplecase studies, with bibliographical research and interviews with producers. In multiple-case studies, the selected production units are distinguished by the adopted production systems, which precludes generalization of their results and provides a basis and a tool for other studies because they are not considered "sampling units" (Ferreira et al., 2014).

Data collection for the calculations was performed in 29 soybean crop areas in the 20142015 crop year. The survey was performed using structured interviews with farmers and/or data obtained from research institutions such as the National Food Supply Company (CONAB), the Federation of Agriculture and Livestock of Goiás (FAEG), the Agriculture Research and Rural Extension Company of Santa Catarina (EPAGRI), and the Center of Social Economy and Agricultural Planning of the School of Agriculture "Luiz de Queiroz" (CEPA-ESALQ) (Table 1). 
Table 1

Location, size, and mean yield of soybean in different regions of Brazil

\begin{tabular}{|c|c|c|c|c|c|c|c|}
\hline Area & City/State & $\begin{array}{l}\text { Size of the } \\
\text { area (ha) }\end{array}$ & $\begin{array}{c}\text { Mean yield } \\
\left(\mathrm{Mg} \mathrm{ha}^{-1}\right)\end{array}$ & Area & City/State & $\begin{array}{l}\text { Size of the } \\
\text { area (ha) }\end{array}$ & $\begin{array}{c}\text { Mean yield } \\
\left(\mathrm{Mg} \mathrm{ha}^{-1}\right)\end{array}$ \\
\hline \multicolumn{4}{|c|}{-------- Midwest region ---------- } & \multicolumn{4}{|c|}{ - Northeast Region - - } \\
\hline 1 & $\begin{array}{l}\text { **Campo N. } \\
\text { Parecis-MT }{ }^{1,6}\end{array}$ & 2000 & 3.12 & 21 & $* *$ Barreiras-BA ${ }^{1,6}$ & 2000 & 3.00 \\
\hline 2 & $\begin{array}{l}* * \text { Campo N. } \\
\text { Parecis-MT }\end{array}$ & 2000 & 3.12 & 22 & $* *$ Balsas-MA ${ }^{1,6}$ & 1000 & 3.12 \\
\hline 3 & $\begin{array}{c}* * \text { Primavera do } \\
\text { Leste-MT } \mathrm{MT}^{1,6}\end{array}$ & 1125 & 3.24 & \multicolumn{4}{|c|}{ 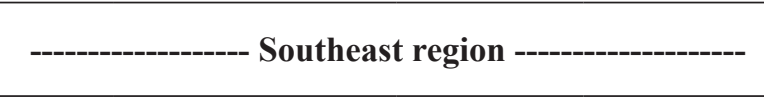 } \\
\hline 4 & $\begin{array}{l}* * \text { Primavera do } \\
\text { Leste-MT }^{1}\end{array}$ & 375 & 3.24 & 23 & **Unaí-MG ${ }^{1,6}$ & 650 & 3.12 \\
\hline 5 & $* *$ Sorriso-MT $\mathrm{T}^{1,6}$ & 1500 & 3.12 & \multicolumn{4}{|c|}{ 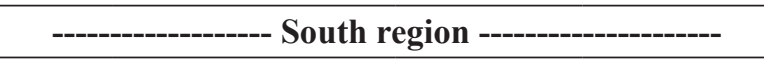 } \\
\hline 6 & $* *$ Sorriso-MT ${ }^{1}$ & 1500 & 3.18 & 24 & $\begin{array}{c}* * \text { EPAGRICEPA- } \\
\text { SC }^{3,6}\end{array}$ & & 3.30 \\
\hline 7 & $* *$ FAEG-GO ${ }^{2,6}$ & & 3.48 & 25 & $\begin{array}{c}\text { **Campo }^{*} \text { Camo-PR } \\
\text { Mourão-PR }\end{array}$ & 40 & 3.00 \\
\hline 8 & $* *$ Rio Verde-GO ${ }^{1,6}$ & 450 & 3.30 & 26 & $* *$ Londrina-PR ${ }^{1,6}$ & 60 & 3.60 \\
\hline 9 & $* *$ Cristalina-GO ${ }^{1,6}$ & 1000 & 3.00 & 27 & **Londrina-PR ${ }^{1}$ & 60 & 3.50 \\
\hline 10 & $*$ Montividiu-GO ${ }^{5,6}$ & 1800 & 3.36 & 28 & $* *$ Cruz Alta-RS ${ }^{1,6}$ & 200 & 2.70 \\
\hline 11 & *Montividiu-GO ${ }^{5,6}$ & 1298 & 2.88 & 29 & $\begin{array}{c}\text { **São Luiz } \\
\text { Gonzaga-RS }\end{array}$ & 35 & 2.20 \\
\hline 12 & *Rio Verde-GO ${ }^{5,6}$ & 400 & 2.28 & & Total: & 20583 & \\
\hline 13 & *Montividiu-GO ${ }^{5,6}$ & 300 & 3.24 & & Mean: & & 3.03 \\
\hline 14 & *Montividiu-GO $\mathrm{GO}^{5,6}$ & 515 & 3.24 & & & & \\
\hline 15 & *Montividiu-GO ${ }^{5,6}$ & 300 & 3.00 & & & & \\
\hline 16 & *Rio Verde-GO $\mathrm{GO}^{5,6}$ & 155 & 1.02 & & & & \\
\hline 17 & $*$ MontividiuGO ${ }^{5,6}$ & 100 & 3.36 & & & & \\
\hline 18 & $*$ Montividiu-GO ${ }^{5,6}$ & 220 & 2.95 & & & & \\
\hline 19 & $* *$ Brasilia-DF $F^{1,6}$ & 500 & 3.10 & & & & \\
\hline 20 & $\begin{array}{l}\text { **Chapadão do } \\
\text { Sul-MS }{ }^{1,6}\end{array}$ & 1000 & 3.10 & & & & \\
\hline
\end{tabular}

${ }^{1}$ Data provided by the National Food Supply Company (CONAB). ${ }^{2}$ Data provided by the Federation of Agriculture and Livestock of Goiás (FAEG). ${ }^{3}$ Data provided by the Agriculture Research and Rural Extension Company of Santa Catarina (EPAGRI). ${ }^{4}$ Center of Social Economy and Agricultural Planning (CEPA). ${ }^{5}$ Data provided by several farmers in the region. ${ }^{6}$ Sowing performed using transgenic seeds. *On-site evaluation, through interviews with producers. **Evaluation via data provided by official bodies in the regions.

The data collected were categorized as follows: a) amount of human labor, fuel, synthetic and organic fertilizers, seeds, seedlings, herbicides, insecticides, fungicides, and other inputs involved from sowing to harvest; b) agricultural operations used in crop management, and tractors, machinery, and/or implements used for these services; c) crop grain yield; and d) technical parameters such as duration of each agricultural operation and fuel consumption $\left(\mathrm{L} \mathrm{h}^{-1}\right)$. The detailed data of the 
soybean areas assessed in this study are described in Table 1.

The data regarding the amount of inputs and the agricultural practices were converted into units of energy by multiplying the physical product by the respective conversion factors, known as energy coefficients, expressed as MJ (Assenheimer et al., 2009; Capellesso \& Cazella, 2013). The energy coefficients used in the present study were based on data from the literature, both for inputs (factors required for production) and outputs (grain production) (Table 2).

Table 2

Main energy coefficients that were used to determine the energy consumption by the analyzed crops

\begin{tabular}{|c|c|c|c|}
\hline \multicolumn{4}{|c|}{ 1. Direct energy } \\
\hline Specifications & Unity & $\begin{array}{l}\text { Energy coef- } \\
\text { ficient (EC) }\end{array}$ & Bibliographic reference \\
\hline Human labor & $\mathrm{MJ} \mathrm{h}^{-1}$ men & 7.84 & $\begin{array}{l}\text { Boddey, Soares, Alves and Urquiaga } \\
\text { (2008) }\end{array}$ \\
\hline Synthetic Nitrogen Fertilizers (N) & $\mathrm{MJ} \mathrm{kg}{ }^{-1}$ & 63.79 & Macedônio and Picchioni (1985) \\
\hline Phosphate Synthetic Fertilizers $\left(\mathrm{P}_{2} \mathrm{O}_{5}\right)$ & $\mathrm{MJ} \mathrm{kg}^{-1}$ & 13.97 & Macedônio and Picchioni (1985) \\
\hline Potash Synthetic Fertilizers $\left(\mathrm{K}_{2} \mathrm{O}\right)$ & $\mathrm{MJ} \mathrm{kg}{ }^{-1}$ & 9.79 & Macedônio and Picchioni (1985) \\
\hline Limestone & MJ kg-1 & 0.167 & Comitre (1993) \\
\hline Plaster & $\mathrm{MJ} \mathrm{kg}{ }^{-1}$ & 0.167 & Vieira (2007) \\
\hline Micronutrient Zinc & $\mathrm{MJ} \mathrm{kg}{ }^{-1}$ & 8.37 & Pimentel (1980) \\
\hline Cobalt + Molybdenum (CoMo) & $\mathrm{MJ} \mathrm{L}^{-1}$ & 0.042 & Gomes (2012) \\
\hline Micronutrients in general & $\mathrm{MJ} \mathrm{kg}^{-1}$ & 6.32 & Souza, Casali, Santos and Cecon (2008) \\
\hline Chicken Bed & MJ kg-1 & 0.126 & Souza et al. (2008) \\
\hline Natural phosphate & $\mathrm{MJ} \mathrm{kg}{ }^{-1}$ & 0.63 & Quadros and Kokuszka (2007) \\
\hline${ }^{1}$ Energy converter & MJ R\$ & 2.23 & Energy Research Office (EPE) (2015) \\
\hline Calorific value of diesel oil & MJ L-1 & 43.93 & Comitre (1993) \\
\hline${ }^{2}$ Calorific value of lubricating oil & MJ L-1 & 35.94 & Comitre (1993) \\
\hline${ }^{2}$ Calorific value of grease & $\mathrm{MJ} \mathrm{L}^{-1}$ & 49.22 & Comitre (1993) \\
\hline Soybean Seeds & $\mathrm{MJ} \mathrm{kg}{ }^{-1}$ & 31.75 & Pimentel (1980) \\
\hline Inoculants & MJ dose $\mathrm{e}^{-1}$ & 5.25 & Sá et al. (2013) \\
\hline${ }^{4}$ Herbicides, insecticides, fungicides, etc ... & $\mathrm{kg}$ ou L/i.a & (Table 3) & Pimentel (1980) \\
\hline \multicolumn{4}{|c|}{ 2. Indirect Energies } \\
\hline 5,6 Tractor or machines (Self Propelled) & $\mathrm{MJ} \mathrm{kg}^{-1}$ & 69.83 & Macedônio and Picchioni (1985) \\
\hline Self-Propelled Harvesters & $\mathrm{MJ} \mathrm{kg}{ }^{-1}$ & 69.87 & Macedônio and Picchioni (1985) \\
\hline Attachments (not self-propelled) & $\mathrm{MJ} \mathrm{kg}^{-1}$ & 57.2 & Macedônio and Picchioni (1985) \\
\hline
\end{tabular}

${ }^{1}$ For very specific inputs and with little significant contribution, the energy value was estimated based on the cost of one monetary unit for the Gross Domestic Product related to the National Energy Balance, both of $2014 .{ }^{2}$ For the calculation of the consumption of lubricating oil, it was considered $1.5 \%$ of diesel consumption and, for grease, $33 \%$ of lubricant consumption. ${ }^{4}$ Varies according to the active ingredient (ia) and formulation used (Table 3). ${ }^{5}$ An energy value for repairing tractors, machines and agricultural implements was also established, which corresponds to $5 \%$ of the total energy used in their manufacture. ${ }^{6} \mathrm{~A}$ value of $2.24 \%$ (Boddey et al., 2008) was also considered in relation to total energy expenditure in agricultural production as an estimate of energy expenditure on the transportation of machinery, implements and inputs to the crop. 
Energy depreciation $(E D)$ and indirect energy associated with tractors, machinery, and/or agricultural implements was calculated using the equation (Beber, 1989):

$$
E D=\frac{0.9 \times M}{S l} \times d u \times E C
$$

Where $M$ is the mass of the tractor or agricultural implement in $\mathrm{kg}, S l$ is the service life of the tractor or agricultural implement in hours, $d u$ is the duration of use in hours, and EC is the energy coefficient of the assessed tractor, machinery or agricultural implement. The masses of the tractors, machinery, and implements were obtained from manufacturers' catalogues. The values of service life were obtained from CONAB (Companhia Nacional de Abastecimento [CONAB], 2010).

The energy coefficients used for the conversion of herbicide, insecticide, and fungicide quantities into energy values were estimated based on the literature (Pimentel, 1980); the amount of energy used with these inputs was thus attributed according to their formulations (Table 3).

Table 3

Energy coefficients used to calculate energy expenditures with herbicides and pesticides for the control of pests and diseases in the crops assessed in this study (Pimentel, 1980)

\begin{tabular}{|c|c|}
\hline Formulation & Energy coefficient of a.i. $\left(\mathrm{MJ} \mathrm{kg}^{-1}\right.$ or $\left.\mathrm{L}^{-1}\right)$ \\
\hline \multicolumn{2}{|c|}{ Herbicides } \\
\hline Dispersible concentrate & 418.3 \\
\hline Soluble powder & 262.8 \\
\hline Pellets & 362.6 \\
\hline *Mean value & 347.9 \\
\hline \multicolumn{2}{|c|}{ Plant protection products for pest control } \\
\hline Dispersible concentrate & 363.9 \\
\hline Soluble powder & 311.1 \\
\hline Granulate & 311.1 \\
\hline Wettable powder & 257.4 \\
\hline *Mean value & 310.8 \\
\hline \multicolumn{2}{|c|}{ Plant protection products for disease control } \\
\hline Dispersible concentrate & 271.8 \\
\hline Soluble powder & 116.3 \\
\hline Granulate & 216.0 \\
\hline Wettable powder & 216.0 \\
\hline Mean value* & 205.0 \\
\hline
\end{tabular}

a.i. - active ingredient. * Mean value attributed by the authors to other types of formulations. 
The calculations of energy consumption in the agricultural operations, including the application of additives and fertilizers, sowing, internal transportation, application of herbicides, insecticides and fungicides, and harvesting were obtained using the fuel used $\left(\mathrm{L} \mathrm{h}^{-1}\right)$ by the tractorimplement system or machine combined with the performance of this system or machine $\left(\mathrm{ha}^{-1}\right)$. Based on these data, the fuel expenditure in $\mathrm{Lh}^{1}$ was divided by the operational yield in ha $\mathrm{h}^{-1}$ to obtain the fuel expenditure in $\mathrm{L} \mathrm{ha}^{-1}$. Energy input (in MJ $\mathrm{ha}^{-1}$ ) was obtained considering the calorific value of diesel oil (47.73 $\mathrm{MJ} \mathrm{L}^{1}$ ). Most of these data were acquired through the field record of the Producers and / or through data provided by official bodies, as described in Table 1.

Some correction factors were used in the calculations of the fuel (diesel) used in operations such as grain transportation and transshipping, water transportation, and tillage. The expenditure of fuel during grain and water transportation operations was divided by the cargo-carrying capacity per hectare. For example, if a tractor water tanker uses $10 \mathrm{~L}$ of diesel to carry $2000 \mathrm{~L}$ of water, and the volume of the solution used is $200 \mathrm{~L} \mathrm{ha}^{1}, 10 \mathrm{~L}$ of diesel is consumed in this operation for each 10 ha, i.e., 1 $\mathrm{L}$ ha ${ }^{1}$ of diesel was used for the transportation of water. Diesel consumption in tillage operations was divided by the number of years of residual effect on the soil from the used management system. In plowing and harrowing operations, for example, a conservative timeframe of 10 years was used for the mean residual effect of these operations because all the areas were under a system of direct seeding.

The list of tractors, machines, and agricultural implements, as well as their operational yields ( $\mathrm{h}$ $\mathrm{ha}^{-1}$ ) and diesel consumptions $\left(\mathrm{L} \mathrm{ha}^{-1}\right.$ year $\left.{ }^{-1}\right)$ in the abovementioned soybean crop areas, are shown in Table 4. The agricultural operations performed on each property are described in Table 5 . 
Table 4

Identification of tractor sets, machines and implements used in the areas (Id), as well as their Power in HP(Pt), and Operational indicator in $\mathrm{h}^{-1} \mathrm{ha}^{-1}(\mathrm{Op})$ and Consumption in $\mathrm{L}$ diesel ha $\mathrm{hear}^{-1}$ (CC)

\begin{tabular}{|c|c|c|c|c|c|c|c|c|c|c|c|}
\hline Id & $* \mathrm{ST}$ & $\mathrm{Pt}$ & $*$ Ip & $\mathrm{Op}$ & $\mathrm{CC}$ & Id & $* \mathrm{ST}$ & $\mathrm{Pt}$ & $*$ Ip & Op & $\mathrm{CC}$ \\
\hline 1 & ${ }^{1}$ Tra & 110 & ${ }^{6} \mathrm{Ss}$ & 0.16 & 1.62 & 61 & ${ }^{1}$ Tra & 217 & ${ }^{27} \mathrm{Se}$ & 0.19 & 4.88 \\
\hline 2 & ${ }^{2} \mathrm{Sps}$ & 125 & & 0.04 & 0.48 & 62 & ${ }^{4} \mathrm{Har}$ & 284 & & 0.18 & 4.93 \\
\hline 3 & ${ }^{1} \mathrm{Tra}$ & 110 & ${ }^{6} \mathrm{Ss}$ & 0.06 & 0.63 & 63 & ${ }^{1} \mathrm{Tra}$ & 217 & ${ }^{28} \mathrm{Tt}$ & 0.46 & 1.18 \\
\hline 4 & ${ }^{3} \mathrm{Spc}$ & 152 & & 0.16 & 2.31 & 64 & Truck & 360 & & 0.8 & 2.87 \\
\hline 5 & ${ }^{1} \mathrm{Tra}$ & 225 & ${ }^{7} \mathrm{Se}$ & 0.26 & 6.05 & 65 & ${ }^{1}$ Tra & 175 & ${ }^{29} \mathrm{Lh}$ & 0.18 & 2.85 \\
\hline 6 & ${ }^{4} \mathrm{Har}$ & 270 & & 0.33 & 8.48 & 66 & ${ }^{1} \mathrm{Tra}$ & 175 & ${ }^{11} \mathrm{Ss}$ & 0.14 & 1.65 \\
\hline 7 & Truck & 110 & & 0.46 & 1.16 & 67 & ${ }^{2} \mathrm{Sps}$ & 245 & & 0.02 & 0.4 \\
\hline 8 & ${ }^{1} \mathrm{Tra}$ & 225 & ${ }^{8} \mathrm{Pd}$ & 0.33 & 8.77 & 68 & ${ }^{1} \mathrm{Tra}$ & 217 & ${ }^{11} \mathrm{Ss}$ & 0.07 & 0.79 \\
\hline 9 & ${ }^{1} \mathrm{Tra}$ & 110 & ${ }^{6} \mathrm{Ss}$ & 0.1 & 1.01 & 69 & ${ }^{1}$ Tra & 335 & ${ }^{30} \mathrm{Se}$ & 0.1 & 3.17 \\
\hline 10 & ${ }^{1} \mathrm{Tra}$ & 110 & ${ }^{9} \mathrm{Ss}$ & 0.08 & 0.47 & 70 & ${ }^{4} \mathrm{Har}$ & 375 & & 0.1 & 3.61 \\
\hline 11 & ${ }^{2} \mathrm{Sps}$ & 128 & & 0.05 & 0.6 & 71 & ${ }^{1} \mathrm{Tra}$ & 217 & ${ }^{13} \mathrm{Tt}$ & 0.46 & 1.18 \\
\hline 12 & ${ }^{1} \mathrm{Tra}$ & 110 & ${ }^{10} \mathrm{Tt}$ & 0.01 & 0.003 & 72 & Truck & 480 & & 0.57 & 1.73 \\
\hline 13 & ${ }^{1} \mathrm{Tra}$ & 110 & ${ }^{11} \mathrm{Ss}$ & 0.1 & 1.18 & 73 & ${ }^{1} \operatorname{Tra}$ & 127 & ${ }^{31} \mathrm{Ss}$ & 0.11 & 1.45 \\
\hline 14 & ${ }^{3} \mathrm{Spc}$ & 152 & & 0.1 & 1.57 & 74 & ${ }^{1} \mathrm{Tra}$ & 127 & ${ }^{31} \mathrm{Ss}$ & 0.04 & 0.54 \\
\hline 15 & ${ }^{1} \mathrm{Tra}$ & 225 & ${ }^{12} \mathrm{Se}$ & 0.25 & 7.73 & 75 & ${ }^{1} \operatorname{Tra}$ & 127 & ${ }^{27} \mathrm{Se}$ & 0.33 & 5.15 \\
\hline 16 & ${ }^{4} \mathrm{Har}$ & 378 & & 0.25 & 8.99 & 76 & ${ }^{4} \mathrm{Har}$ & 270 & & 0.17 & 4.46 \\
\hline 17 & ${ }^{1} \mathrm{Tra}$ & 110 & ${ }^{11} \mathrm{Ss}$ & 0.16 & 1.9 & 77 & ${ }^{1} \operatorname{Tra}$ & 95 & ${ }^{32} \mathrm{Tt}$ & 0.16 & 0.27 \\
\hline 18 & ${ }^{2} \mathrm{Sps}$ & 245 & & 0.03 & 0.77 & 78 & Truck & 440 & & 0.41 & 0.48 \\
\hline 19 & ${ }^{1} \mathrm{Tra}$ & 110 & ${ }^{11} \mathrm{Ss}$ & 0.08 & 0.95 & 79 & ${ }^{1} \mathrm{Tra}$ & 86 & ${ }^{9} \mathrm{Ss}$ & 0.28 & 2.05 \\
\hline 20 & ${ }^{3} \mathrm{Spc}$ & 152 & & 0.12 & 1.88 & 80 & ${ }^{1} \mathrm{Tra}$ & 86 & ${ }^{14} \mathrm{Sp}$ & 0.14 & 1.14 \\
\hline 21 & ${ }^{1} \mathrm{Tra}$ & 225 & ${ }^{7} \mathrm{Se}$ & 0.12 & 2.76 & 81 & ${ }^{1} \mathrm{Tra}$ & 68 & ${ }^{15} \mathrm{Tt}$ & 3.33 & 1.45 \\
\hline 22 & ${ }^{4} \mathrm{Har}$ & 378 & & 0.2 & 7.19 & 82 & Tractor & 127 & ${ }^{33} \mathrm{Se}$ & 0.3 & 3.67 \\
\hline 23 & ${ }^{1} \mathrm{Tra}$ & 225 & ${ }^{13} \mathrm{Tt}$ & 0.2 & 0.47 & 83 & ${ }^{4} \mathrm{Har}$ & 270 & & 0.48 & 12.23 \\
\hline 24 & Ssp & 250 & & 1 & 2.74 & 84 & ${ }^{1} \mathrm{Tra}$ & 127 & ${ }^{34} \mathrm{Tt}$ & 0.46 & 2.53 \\
\hline 25 & ${ }^{1} \mathrm{Tra}$ & 68 & ${ }^{14} \mathrm{Sp}$ & 0.2 & 1.62 & 85 & truck & 110 & & 0.26 & 0.046 \\
\hline 26 & ${ }^{1} \mathrm{Tra}$ & 68 & ${ }^{15} \mathrm{Tt}$ & 0.5 & 0.27 & 86 & ${ }^{1} \mathrm{Tra}$ & 127 & ${ }^{35} \mathrm{Se}$ & 0.22 & 2.98 \\
\hline 27 & ${ }^{1} \mathrm{Tra}$ & 105 & ${ }^{16} \mathrm{Se}$ & 0.7 & 8.73 & 87 & ${ }^{4} \mathrm{Har}$ & 196 & & 0.14 & 2.7 \\
\hline 28 & ${ }^{4} \mathrm{Har}$ & 196 & & 0.5 & 11.68 & 88 & ${ }^{1} \mathrm{Tra}$ & 127 & ${ }^{36} \mathrm{Tt}$ & 0.33 & 1.01 \\
\hline 29 & ${ }^{1} \mathrm{Tra}$ & 110 & ${ }^{17} \mathrm{Ss}$ & 0.4 & 5.23 & 89 & ${ }^{1}$ Tra & 127 & ${ }^{37} \mathrm{Lh}$ & 0.48 & 6.57 \\
\hline 30 & ${ }^{1} \mathrm{Tra}$ & 110 & ${ }^{14} \mathrm{Sp}$ & 0.42 & 5.47 & 90 & ${ }^{1} \mathrm{Tra}$ & 127 & ${ }^{38} \mathrm{Ss}$ & 0.17 & 2.18 \\
\hline 31 & ${ }^{1} \mathrm{Tra}$ & 110 & ${ }^{16} \mathrm{Se}$ & 1 & 5.04 & 91 & ${ }^{1} \mathrm{Tra}$ & 86 & ${ }^{39} \mathrm{Sp}$ & 0.12 & 0.98 \\
\hline 32 & ${ }^{4} \mathrm{Har}$ & 196 & & 0.7 & 8.03 & 92 & ${ }^{1} \mathrm{Tra}$ & 127 & ${ }^{38} \mathrm{Ss}$ & 0.08 & 1.09 \\
\hline 33 & Truck & 110 & & 0.46 & 1.56 & 93 & Truck & 345 & & 0.8 & 2.47 \\
\hline 34 & ${ }^{1} \mathrm{Tra}$ & 75 & ${ }^{18} \mathrm{Ss}$ & 0.21 & 1.54 & 94 & ${ }^{1} \mathrm{Tra}$ & 165 & ${ }^{32} \mathrm{Tp}$ & 0.25 & 1.26 \\
\hline 35 & ${ }^{2} \mathrm{Sps}$ & 128 & & 0.1 & 1.21 & 95 & ${ }^{1} \mathrm{Tra}$ & 127 & ${ }^{23} \mathrm{Ss}$ & 0.11 & 1.32 \\
\hline 36 & ${ }^{1} \mathrm{Tra}$ & 120 & ${ }^{19} \mathrm{Se}$ & 0.4 & 3.93 & 96 & ${ }^{1} \mathrm{Tra}$ & 75 & ${ }^{14} \mathrm{Sp}$ & 0.06 & 0.41 \\
\hline 37 & ${ }^{1} \mathrm{Tra}$ & 75 & ${ }^{15} \mathrm{Tt}$ & 0.33 & 0.14 & 97 & ${ }^{4} \mathrm{Har}$ & 270 & & 0.24 & 6.2 \\
\hline 38 & ${ }^{4} \mathrm{Har}$ & 175 & & 0.33 & 5.49 & 98 & Truck & 130 & & 0.14 & 0.3 \\
\hline
\end{tabular}


continuation

\begin{tabular}{|c|c|c|c|c|c|c|c|c|c|c|c|}
\hline 39 & Truck & 110 & & 0.46 & 1 & 99 & ${ }^{1} \mathrm{Tra}$ & 145 & ${ }^{40} \mathrm{Ss}$ & 0.1 & 1.42 \\
\hline 40 & ${ }^{1} \mathrm{Tra}$ & 75 & ${ }^{20} \mathrm{Sp}$ & 0.12 & 0.85 & 100 & ${ }^{2} \mathrm{Sps}$ & 165 & & 0.04 & 0.62 \\
\hline 41 & ${ }^{4} \mathrm{Har}$ & 196 & & 0.47 & 8.81 & 101 & ${ }^{4} \mathrm{Har}$ & 284 & & 0.25 & 6.75 \\
\hline 42 & Truck & 110 & & 0.46 & 0.82 & 102 & ${ }^{2} \mathrm{Sps}$ & 128 & & 0.08 & 0.97 \\
\hline 43 & ${ }^{1} \mathrm{Tra}$ & 127 & ${ }^{17} \mathrm{Ss}$ & 0.2 & 3.03 & 103 & ${ }^{1} \operatorname{Tra}$ & 123 & ${ }^{16} \mathrm{Se}$ & 1 & 5.98 \\
\hline 44 & ${ }^{1} \mathrm{Tra}$ & 86 & ${ }^{14} \mathrm{Sp}$ & 0.4 & 4.12 & 104 & ${ }^{1} \mathrm{Tra}$ & 86 & ${ }^{21} \mathrm{Hs}$ & 0.16 & 1.14 \\
\hline 45 & ${ }^{1} \mathrm{Tra}$ & 127 & ${ }^{16} \mathrm{Se}$ & 0.5 & 7.58 & 105 & ${ }^{1} \operatorname{Tra}$ & 175 & ${ }^{41} \mathrm{Se}$ & 0.66 & 5.44 \\
\hline 46 & ${ }^{4} \mathrm{Har}$ & 270 & & 1 & 8.03 & 106 & ${ }^{4} \mathrm{Har}$ & 270 & & 0.4 & 10.28 \\
\hline 47 & ${ }^{1} \mathrm{Tra}$ & 86 & ${ }^{20} \mathrm{Tp}$ & 0.5 & 5.14 & 107 & ${ }^{1} \operatorname{Tra}$ & 68 & ${ }^{17} \mathrm{Ss}$ & 0.6 & 4.9 \\
\hline 48 & ${ }^{1} \mathrm{Tra}$ & 110 & ${ }^{11} \mathrm{Ss}$ & 0.03 & 0.39 & 108 & ${ }^{1} \mathrm{Tra}$ & 68 & ${ }^{14} \mathrm{Sp}$ & 0.6 & 4.9 \\
\hline 49 & ${ }^{1} \mathrm{Tra}$ & 110 & ${ }^{21} \mathrm{Hs}$ & 0.25 & 2.38 & 109 & ${ }^{4} \mathrm{Har}$ & 270 & & 1 & 9 \\
\hline 50 & ${ }^{1}$ Tra & 145 & ${ }^{22} \mathrm{Se}$ & 0.25 & 4.75 & 110 & ${ }^{1}$ Tra & 217 & ${ }^{26} \mathrm{Pl}$ & 1 & 9.51 \\
\hline 51 & ${ }^{4} \mathrm{Har}$ & 270 & & 0.37 & 9.51 & 111 & ${ }^{1} \mathrm{Tra}$ & 180 & ${ }^{40} \mathrm{Ss}$ & 0.5 & 7.14 \\
\hline 52 & ${ }^{1}$ Tra & 100 & ${ }^{23} \mathrm{Ss}$ & 0.33 & 4.71 & 112 & ${ }^{2} \mathrm{Sps}$ & 128 & & 0.13 & 1.52 \\
\hline 53 & ${ }^{1}$ Tra & 100 & ${ }^{14} \mathrm{Sp}$ & 0.2 & 1.9 & 113 & ${ }^{1} \mathrm{Tra}$ & 180 & ${ }^{41} \mathrm{Se}$ & 0.5 & 9.81 \\
\hline 54 & ${ }^{1} \operatorname{Tra}$ & 140 & ${ }^{21} \mathrm{Hs}$ & 0.03 & 0.28 & 114 & ${ }^{1} \mathrm{Tra}$ & 88 & ${ }^{42} \mathrm{Ss}$ & 0.13 & 1.12 \\
\hline 55 & ${ }^{1}$ Tra & 140 & ${ }^{24} \mathrm{Se}$ & 0.6 & 9.19 & 115 & ${ }^{4} \mathrm{Har}$ & 238 & & 0.6 & 7.54 \\
\hline 56 & ${ }^{4} \mathrm{Har}$ & 175 & & 0.19 & 3.24 & 116 & ${ }^{1}$ Tra & 225 & ${ }^{29} \mathrm{Lh}$ & 0.17 & 2.34 \\
\hline 57 & ${ }^{1} \mathrm{Tra}$ & 217 & ${ }^{25} \mathrm{Sp}$ & 0.73 & 16.06 & 117 & ${ }^{2} \mathrm{Sps}$ & 245 & & 0.05 & 1.16 \\
\hline 58 & ${ }^{1}$ Tra & 217 & ${ }^{26} \mathrm{Pl}$ & 0.56 & 10.46 & 118 & ${ }^{1}$ Tra & 110 & ${ }^{17} \mathrm{Ss}$ & 0.13 & 0.32 \\
\hline 59 & ${ }^{2} \mathrm{Sps}$ & 128 & & 0.03 & 0.31 & & & & & & \\
\hline
\end{tabular}

${ }^{*} \mathrm{ST}$ (Source of traction), Ip (Implements). ${ }^{* *} \mathrm{PR}$ (required power). ${ }^{1} \mathrm{Tra}$ (Trator), ${ }^{2} \mathrm{Sps}$ (Self-propelled sprayer), ${ }^{3} \mathrm{Spc}($ Self propelled crawler excavator), ${ }^{4} \mathrm{Har}$ (Harvester), ${ }^{5} \mathrm{Ssp}$ (Solids Spreader selfp ropelled), ${ }^{6} \mathrm{Ss}$ (Solids spreader $\left(2-3 \mathrm{~m}^{-3}\right)$, ${ }^{7} \mathrm{Se}$ (Seeder PR $\left.193 \mathrm{cv}\right)$, ${ }^{8} \mathrm{Pd}$ (Plow 26 discs), ${ }^{9} \mathrm{Ss}$ (Solids spreader up to $1 \mathrm{~m}^{-3}$ ), ${ }^{10} \mathrm{Tt}$ (Tank trailer 4000 a $\left.5000 \mathrm{~L}\right),{ }^{11} \mathrm{Ss}$ (Spreader Stara Hércules 10000$),{ }^{12} \mathrm{Se}$ (Seeder John Deere 2117), ${ }^{13} \mathrm{Tt}$ (Trailer Stara Reboke Ninja 25000), ${ }^{14} \mathrm{Sp}$ (Sprayer $\left.2000 \mathrm{~L}\right),{ }^{15} \mathrm{Tt}$ (Tank trailer $\left.3000 \mathrm{~L}\right),{ }^{16} \mathrm{Se}(\mathrm{Seeder}$ PR $99 \mathrm{cv}$ ), ${ }^{17} \mathrm{Ss}$ (Solids spreader 4 to $6 \mathrm{~m}^{-3}$ ), ${ }^{18} \mathrm{Ss}$ (Solids spreader 1 to $2 \mathrm{~m}^{-3}$ ), ${ }^{19} \mathrm{Se}$ (Seeder PR $82 \mathrm{cv}$ ), ${ }^{20} \mathrm{Sp}(\mathrm{Sprayer} 600 \mathrm{~L}),{ }^{20} \mathrm{Tp}$ (Transshipping trailer $2 \mathrm{~m}^{3}$ ), ${ }^{21} \mathrm{Hs}$ (Hydraulic shovel Stara), ${ }^{22} \mathrm{Se}$ (Seeder JM7080 PD Guerra), ${ }^{23} \mathrm{Ss}$ (Spreader Stara Bruttus 6000), ${ }^{24} \mathrm{Se}$ (Seeder PR $\left.128 \mathrm{cv}\right),{ }^{25} \mathrm{Sp}$ (Subsoiler plow 9 shanks), ${ }^{26} \mathrm{Pl}$ (Plow 18 discs), ${ }^{27} \mathrm{Se}$ (Seeder Jumil JM3090), ${ }^{28} \mathrm{Tt}$ (Transshipping trailer JAN Tanker 25000), ${ }^{29} \mathrm{Lh}$ (Levelling harrow 48 discs), ${ }^{30} \mathrm{Se}$ (Seeder John Deere DB50), ${ }^{31}$ Ss (Spreader Jan Lancer 12000 $\mathrm{TM}$ ), ${ }^{32} \mathrm{Tt}$ (Transshipping trailer 8 to $10 \mathrm{~m}^{-3}$ ), ${ }^{33} \mathrm{Se}$ (Seeder Tatu Marchesan PST DUO), ${ }^{34} \mathrm{Tt}$ (Trailer JAN Tanker 10.000), ${ }^{35} \mathrm{Se}$ (Seeder Tatu Marchesan Ultra Flex Suprema), ${ }^{36} \mathrm{Tt}$ (Transshipping trailer JAN Tanker Polietileno 17000), ${ }^{37} \mathrm{Lh}$ (Levelling harrow 42 discs), ${ }^{38} \mathrm{Ss}$ (Spreader Jumil Precisa), ${ }^{39} \mathrm{Sp}$ (Sprayer $\left.3000 \mathrm{~L}\right),{ }^{40} \mathrm{Ss}$ (Spreader Stara Hércules 15000), ${ }^{41} \mathrm{Se}$ (Seeder PR $\left.143 \mathrm{cv}\right),{ }^{42} \mathrm{Ss}$ (Spreader Stara Twister 1500 APS).

The number of hours worked and total diesel consumption for all these operations, as well as the quantities of seeds, herbicides and pesticides used are listed below in Table 6 . 


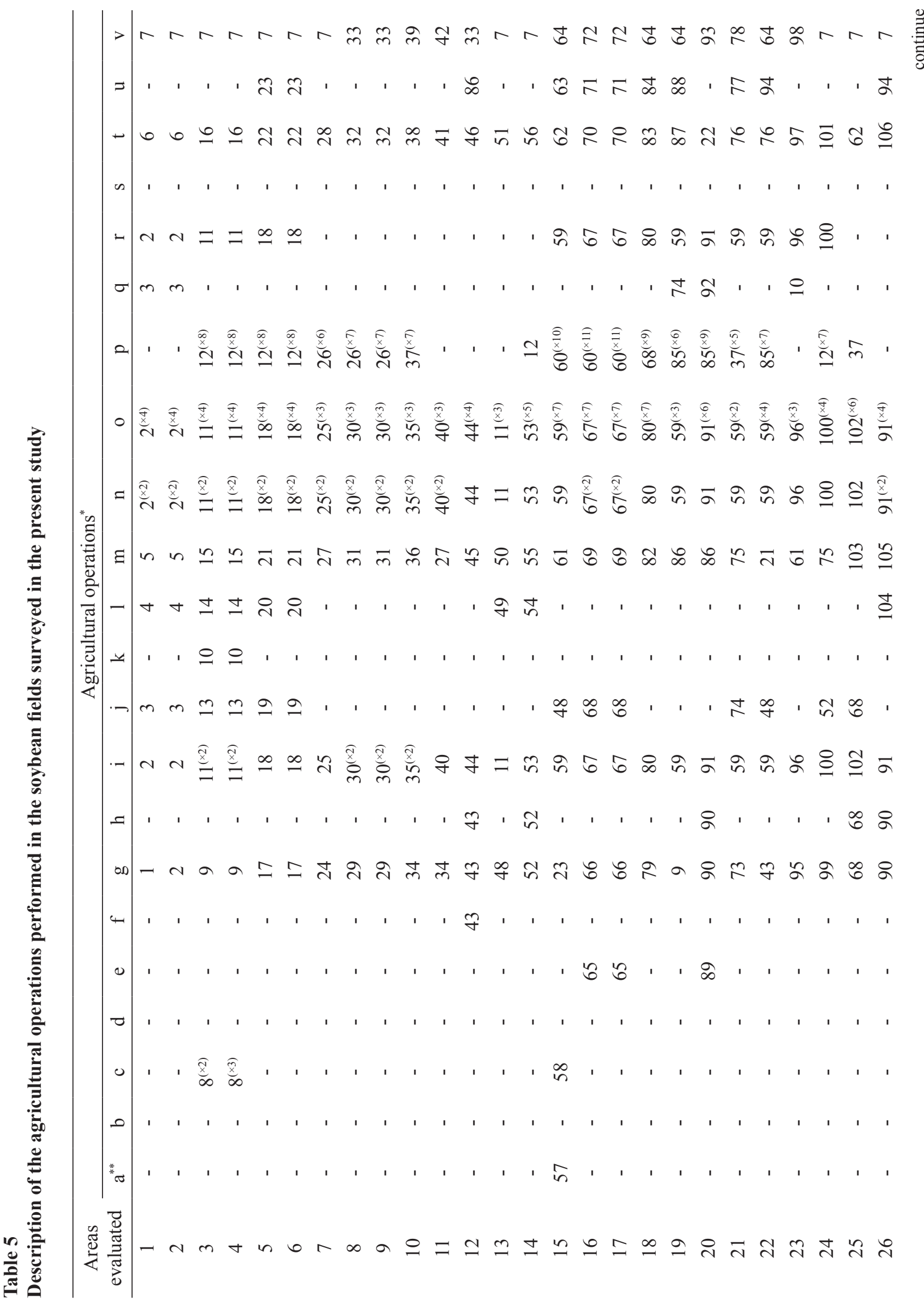




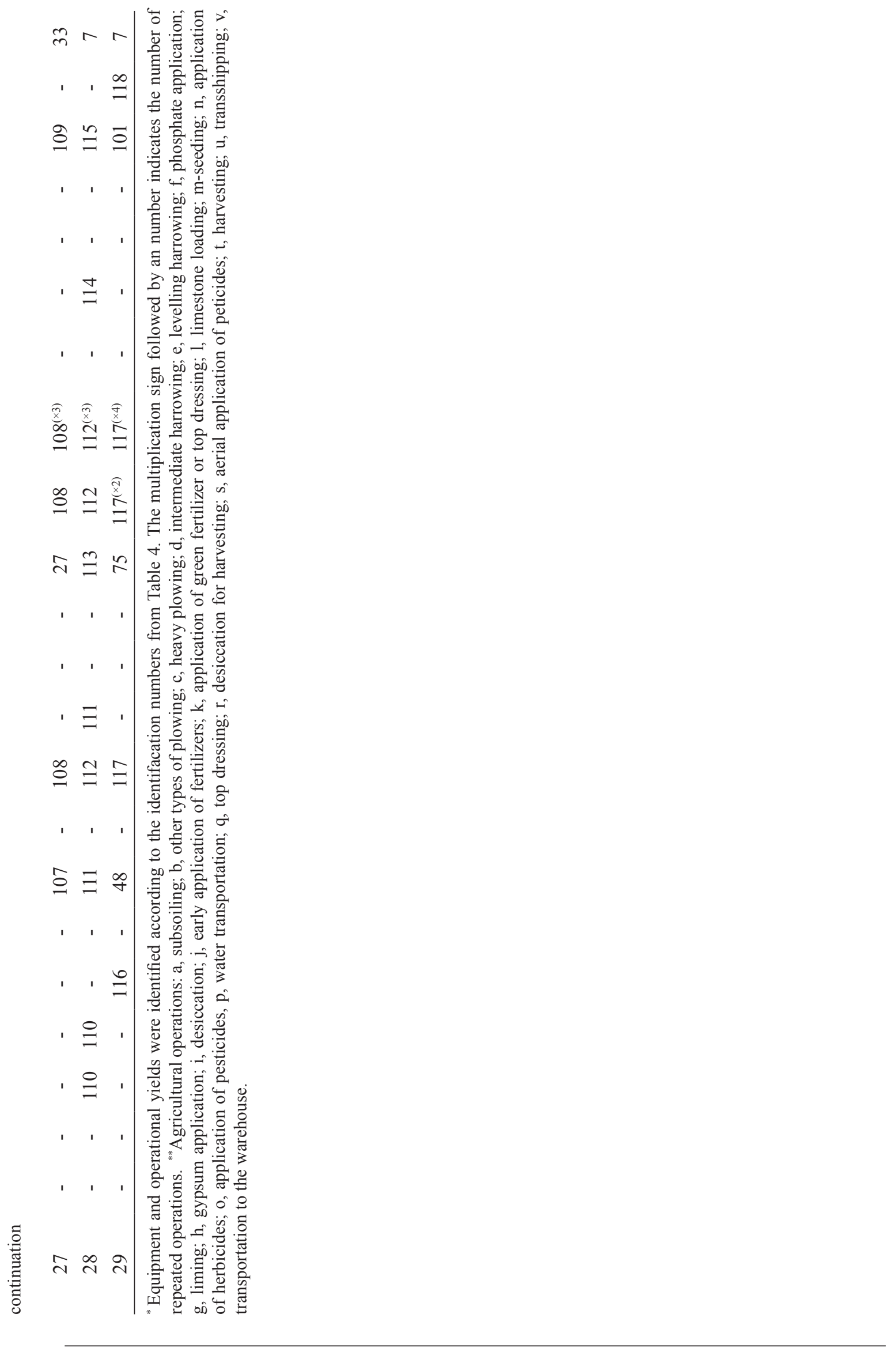

Semina: Ciências Agrárias, Londrina, v. 41, n. 6, suplemento 2, p. 2991-3010, 2020 
Table 6

Quantities of seeds, herbicides, insecticides, fungicides, fuel and human labor used in the areas

\begin{tabular}{|c|c|c|c|c|c|c|}
\hline \multirow{2}{*}{ City / State } & Seeds & Herbicides & Insecticides & Fungicides & \multirow{2}{*}{$\begin{array}{c}\text { Fuel } \\
\text { L ha }^{-1} \text { year }^{-1}\end{array}$} & \multirow{2}{*}{$\frac{\text { Mão-de-obra humana }}{\mathrm{h} \mathrm{ha}^{-1} \text { year }^{-1}}$} \\
\hline & \multicolumn{4}{|c|}{-------- kg ha' year-1 $^{-1------}$} & & \\
\hline Campo N. Parecis-MT & 50 & 5.66 & 1.52 & 0.42 & 23.7 & 1.7 \\
\hline Campo N. Parecis-MT & 50 & 3.44 & 1.52 & 0.42 & 23.7 & 1.7 \\
\hline Primavera do Leste-MT & 66 & 4.58 & 2.98 & 0.84 & 30.4 & 2.1 \\
\hline Primavera do Leste-MT & 66 & 2.46 & 0.15 & 0.84 & 30.4 & 2.1 \\
\hline Sorriso-MT & 45 & 7.52 & 0.77 & 0.38 & 22.7 & 1.6 \\
\hline Sorriso-MT & 45 & 2.76 & 0.77 & 0.38 & 22.7 & 1.6 \\
\hline Campo Mourão-PR & 50 & 3.09 & 0.71 & 0.25 & 31.1 & 4.2 \\
\hline Londrina-PR & 60 & 6.6 & 2.61 & 0.6 & 53.6 & 7.2 \\
\hline Londrina-PR & 60 & 4.25 & 1.83 & 0.3 & 53.6 & 7.2 \\
\hline Cruz Alta-RS & 50 & 3.79 & 0.38 & 0.5 & 21.3 & 3.1 \\
\hline São Luiz Gonzaga-RS & 50 & 4.00 & 0.79 & 0.18 & 23.9 & 2.6 \\
\hline FAEG-GO & 65 & 2.76 & 0.51 & 0.73 & 46.5 & 7.3 \\
\hline Rio Verde-GO & 70 & 3.4 & 1.82 & 0.95 & 21.0 & 1.9 \\
\hline Cristalina-GO & 65 & 3.57 & 0.28 & 0.25 & 35.4 & 3.4 \\
\hline Montividiu-GO & 80 & 3.68 & 2.16 & 0.32 & 15.7 & 3.0 \\
\hline Montividiu-GO & 60 & 3.36 & 2.16 & 0.66 & 14.0 & 2.8 \\
\hline Rio Verde-GO & 60 & 3.36 & 2.16 & 0.66 & 14.0 & 2.8 \\
\hline Montividiu-GO & 60 & 3.7 & 2.15 & 0.32 & 30.3 & 9.9 \\
\hline Montividiu-GO & 70 & 3.55 & 1.42 & 0.75 & 9.5 & 2.2 \\
\hline Montividiu-GO & 50 & 3.9 & 0.41 & 0.66 & 23.8 & 3.3 \\
\hline Rio Verde-GO & 50 & 2.52 & 1.98 & 0.41 & 13.9 & 2.2 \\
\hline Montividiu-GO & 80 & 2.93 & 1.02 & 0.67 & 11.3 & 1.8 \\
\hline Montividiu-GO & 90 & 3.08 & 1.38 & 0.97 & 14.2 & 1.4 \\
\hline Barreiras-BA & 35 & 1.77 & 0.64 & 0.61 & 23.1 & 1.9 \\
\hline Brasilia-DF & 50 & 2.49 & 0.13 & 0.63 & 21.1 & 2.7 \\
\hline Unaí-MG & 45 & 2.71 & 1.61 & 1.36 & 23.3 & 2.7 \\
\hline EPAGRI-CEPA-SC & 55 & 1.68 & 0.06 & 0.17 & 42.8 & 6.6 \\
\hline Balsas-MA & 45 & 3.14 & 0.39 & 0.66 & 62.1 & 5.9 \\
\hline Chapadão do Sul-MS & 70 & 4.27 & 1.16 & 0.25 & 23.3 & 1.6 \\
\hline Mean & 58 & 3.6 & 1.2 & 0.6 & 27.0 & 3.4 \\
\hline
\end{tabular}

The amounts of nitrogen, phosphorous, from grain was $16.74 \mathrm{MJ} \mathrm{kg}^{-1}$ for soybean (Santos potassium, limestone, and gypsum that were applied are listed in Table 7, respectively.

To standardize the calculations of energy output, it was assumed that the mass of grain contained $13 \%$ moisture and that the yield of energy (output) \& Fontaneli, 2007). Thus, to calculate the total energy outputs from agrosystems, grain yield was multiplied by the energy coefficient. The harvest residues were not considered outputs because they are reincorporated into the system (Capellesso \& Cazella, 2013). 
Lastly, EE was calculated by dividing the output energy $\left(\mathrm{MJ} \mathrm{ha}^{-1}\right)$ by the energy input $\left(\mathrm{MJ} \mathrm{ha}^{-1}\right)$ in each production unit; the EB was the result of the difference between the output energy $\left(\mathrm{MJ} \mathrm{ha}^{-1}\right)$ and the input energy $\left(\mathrm{MJ} \mathrm{ha}^{-1}\right)$ (Santos, Fontaneli, Spera,
\& Dreon, 2013). The descriptive statistical analysis to obtain the means, minimum and maximum values, and standard deviation was based on the estimation of EE obtained from each data collection in the 29 soybean crop areas.

Table 7

Amounts of limestone, gypsum, macro- and micronutrients applied in the soybean fields surveyed in the present study

\begin{tabular}{|c|c|c|c|c|c|c|c|c|c|}
\hline \multirow{2}{*}{ City/State } & \multicolumn{9}{|c|}{ Rates $\left(\mathrm{kg} \mathrm{ha}^{-1} \mathrm{yr}^{-1}\right)$} \\
\hline & $\mathrm{N}$ & $\mathrm{P}^{*}$ & $\mathrm{~K}^{*}$ & Limestone & Gypsum & $\mathrm{B}$ & $\mathrm{Zn}$ & $\mathrm{Mn}$ & $\mathrm{Co}+\mathrm{Mo}$ \\
\hline \multicolumn{10}{|l|}{ Soybean } \\
\hline Campo N. Parecis-MT & 12 & 96 & 96 & 375 & - & - & - & 0.49 & 0.08 \\
\hline Campo N. Parecis-MT & 12 & 96 & 96 & 375 & - & - & - & 0.49 & 0.08 \\
\hline Primavera do Leste-MT & - & 105 & 90 & 400 & - & - & - & 0.28 & 0.06 \\
\hline Primavera do Leste-MT & - & 105 & 90 & 400 & - & - & - & 0.28 & 0.06 \\
\hline Sorriso-MT & - & 90 & 90 & 250 & - & - & - & 0.06 & 0.08 \\
\hline Sorriso-MT & - & 90 & 90 & 250 & - & - & - & 0.06 & 0.08 \\
\hline Campo Mourão-PR & 5 & 50 & 45 & 250 & - & - & - & - & - \\
\hline Londrina-PR & - & 50 & 50 & 250 & - & - & - & - & - \\
\hline Londrina-PR & - & 50 & 50 & 250 & - & - & - & - & - \\
\hline Cruz Alta-RS & 5 & 50 & 50 & 250 & - & - & - & - & 0.10 \\
\hline São Luiz Gonzaga-RS & - & 40 & 40 & 250 & - & - & - & - & 0.10 \\
\hline FAEG-GO & 8 & 80 & 72 & 300 & 300 & 1.00 & 10.0 & 1.00 & - \\
\hline Rio Verde-GO & 8 & 80 & 72 & 250 & - & 1.00 & 10.0 & 1.00 & - \\
\hline Cristalina-GO & 8 & 92 & 72 & 250 & 200 & - & - & - & - \\
\hline Montividiu-GO & - & 80 & 96 & 650 & - & - & - & - & - \\
\hline Montividiu-GO & 9 & 90 & 81 & 361 & - & 0.34 & 0.7 & 1.63 & 0.10 \\
\hline Rio Verde-GO & 9 & 90 & 81 & 361 & - & 0.34 & 0.7 & 1.63 & 0.10 \\
\hline Montividiu-GO & 8 & 80 & 72 & 650 & - & - & - & - & - \\
\hline Montividiu-GO & 8 & 90 & 80 & 250 & - & - & - & - & - \\
\hline Montividiu-GO & - & 85 & 92 & 333 & 125 & - & - & - & - \\
\hline Rio Verde-GO & 15 & 150 & 150 & 225 & - & - & - & - & - \\
\hline Montividiu-GO & 8 & 100 & 100 & 250 & - & - & - & - & - \\
\hline Montividiu-GO & 6 & 102 & 30 & 333 & - & - & - & - & - \\
\hline Barreiras-BA & - & 84 & 108 & 250 & - & - & - & - & 0.06 \\
\hline Brasilia-DF & 13 & 93 & 60 & 250 & 175 & - & 0.9 & - & 0.06 \\
\hline Unaí-MG & - & 100 & 90 & 250 & 250 & - & 0.7 & - & 0.07 \\
\hline EPAGRI-CEPA-SC & - & 60 & 90 & 250 & - & - & - & - & - \\
\hline Balsas-MA & - & 182 & 200 & 750 & - & - & - & - & 0.10 \\
\hline Chapadão do Sul-MS & 7 & 70 & 190 & 313 & - & - & - & - & 0.10 \\
\hline Mean & 9 & 87 & 87 & 330 & 210 & 0.70 & 3.8 & 0.70 & 0.10 \\
\hline
\end{tabular}




\section{Results and Discussion}

An average EE of $6.1 ; 6.7 ; 7.1$ and 7.2 respectively in the areas assessed in the Midwest, northeast, southeast and south regions of Brazil
(Table 8). This means that for each MJ of energy consumed, these aforementioned values of energy were produced in the form of grain (Table 8).

Table 8

Total energy consumed (MJ ha-1) (TEC), total energy produced (MJ ha-1) (TEP) (b) and energy efficiency (EE) in the agricultural stage of soybean areas evaluated

\begin{tabular}{|c|c|c|c|c|c|c|c|}
\hline Area & TEC $\left(\mathrm{MJ} \mathrm{ha}^{-1}\right)$ & TEP $\left(\mathrm{MJ} \mathrm{ha}^{-1}\right)$ & $\mathrm{EE}$ & Area & TEC $\left(\mathrm{MJ} \mathrm{ha}^{-1}\right)$ & TEP $\left(\mathrm{MJ} \mathrm{ha}^{-1}\right)$ & $\mathrm{EE}$ \\
\hline \multicolumn{4}{|c|}{----------- Midwest region ----------- } & \multicolumn{4}{|c|}{------------ Northeast Region ----------- } \\
\hline 1 & 9208.7 & 52228.8 & 5.7 & 21 & 5890.8 & 50220.0 & 8.5 \\
\hline 2 & 8167.6 & 52228.8 & 6.4 & 22 & 10986.9 & 52228.8 & 4.8 \\
\hline 3 & 9998.2 & 54237.6 & 5.4 & Mean: & 8438.9 & 51224.4 & 6.7 \\
\hline 4 & 9323.5 & 54237.6 & 5.8 & \multicolumn{4}{|c|}{-------- Southeast region ------------ } \\
\hline 5 & 8735.4 & 52228.8 & 6.0 & 23 & 7383.5 & 52228.8 & 7.1 \\
\hline 6 & 6157.1 & 53233.2 & 8.7 & \multicolumn{4}{|c|}{------------- South region ---------------- } \\
\hline 7 & 8589.4 & 58255.2 & 6.9 & 24 & 6793.9 & 55242.0 & 8.3 \\
\hline 8 & 8046.6 & 55242.0 & 6.9 & 25 & 6419.2 & 50220.0 & 8.0 \\
\hline 9 & 8458.3 & 50220.0 & 6.0 & 26 & 8857.8 & 60264.0 & 7.0 \\
\hline 10 & 8615.6 & 56246.4 & 6.5 & 27 & 8533.1 & 58590.0 & 7.0 \\
\hline 11 & 8302.5 & 48211.2 & 5.8 & 28 & 6411.6 & 45198.0 & 7.0 \\
\hline 12 & 8302.5 & 38167.2 & 4.6 & 29 & 6092.0 & 36828.0 & 6.1 \\
\hline 13 & 9452.1 & 54254.3 & 5.7 & Mean: & 7184.6 & 51057.0 & 7.2 \\
\hline 14 & 7826.0 & 54237.6 & 6.9 & $\begin{array}{l}\text { Overall } \\
\text { average: }\end{array}$ & 8145.4 & 50722.2 & 6.4 \\
\hline 15 & 7404.4 & 50220.0 & 6.8 & & & & \\
\hline 16 & 9116.1 & 17074.8 & 1.9 & & & & \\
\hline 17 & 8145.4 & 56246.4 & 6.9 & & & & \\
\hline 18 & 8593.2 & 49366.3 & 5.7 & & & & \\
\hline 19 & 7026.6 & 51894.0 & 7.4 & & & & \\
\hline 20 & 9379.5 & 51894 & 5.5 & & & & \\
\hline Mean: & 8442.4 & 50496.2 & 6.1 & & & & \\
\hline
\end{tabular}

These results of EE regarding the 2014-2015 crop year were lower than those found in the literature: 7.8 (Assenheimer et al., 2009), 9.80 (Santos et al., 2011), 13.66 (Ferreira et al., 2014) and 18.64 (A. T. Campos et al., 2009). This difference is explained by differences in crop management and grain yields among the assessed areas and between the crop years, and also by the fact that those studies included a small number of areas in specific regions. Thus, the various areas assessed in the different regions included in the present study were under different edaphoclimatic conditions and technological packages that together interfered with the energy inputs and outputs within the production system of this crop. Even with this greater representativeness of edaphoclimatic conditions and crop management, 
the EE of the different regions assessed obtained in the present study was higher than the EEs found in some other studies (4.27 and 5.30) (Mourad \& Walter, 2011; Riquetti, 2014).

In addition, the yield of soybean in the 20142015 crop year was below average, i.e., there was a lower energy output in the form of grains because of the influence of a severe drought that occurred in the central-south region of Brazil (CONAB, 2014). These low grain yields can be seen, for example, in the areas of Sorriso-MT, Rio Verde-GO, BrasiliaDF, Chapadão do Sul-MS, Campo Mourão-PR and Cruz Alta-RS (Table 1).

The areas evaluated within the Midwest region of Brazil, showed great variations in $\mathrm{EE}$ due to climatic influence and, as a consequence, the management adopted. This influence is confirmed by the lower EE (1.9) obtained in the present study in an area of Rio Verde-GO (Table 8). Problems caused by the lack of rain in this area led to a $67 \%$ lower productivity than the mean obtained in the remaining assessed areas, which drastically affected its EE. On the other hand, the highest EE (8.7) was obtained in the areas of Sorriso-MT (Table 8). This result is a reflection of the characteristics of grain production in this region, which is the major soybean-producing region of Brazil. The main factors contributing to the high EE of Sorriso-MT, relative to the other areas, are the lower energy expenditures on seeds and herbicides and a good yield response associated with a high investment in highly energy-efficient technologies such as: (i) machinery and implements with a high operational yield and (ii) frequent monitoring of the level of soil fertility and of pests, diseases, and invasive weeds, which reduced excessive use of inputs. This wide variation in EE (1.9 - 8.7) within the same crop year demonstrates the relevance of determining EE in different regions that are representative of the soybean crop.
The two areas assessed in the northeast region also showed a $44 \%$ difference in the $\mathrm{EE}$ value between the areas, and this was mainly due to the difference in management between the areas, as the Balsas-MA area had a higher energy expenditure with mechanization and fuel in relation to the area located in Barreiras-BA (Tables 8 and 9).

In the southeast region, it was only possible to evaluate an area in the Unaí-MG region. The main characteristics of this area, which resulted in an EE of 7.1 (Table 8), were productivity below the national average (3.12 $\mathrm{Mg} \mathrm{ha}^{-1}$ ) (Table 1) and main energy expenditures with fertilizers, seeds and herbicides (Table 9). As occurred in the Midwest region, in this area evaluated in the southeast region, fertilizers were responsible for the largest expense, followed by seeds and herbicides (Table 9).

In the southern region of Brazil, on the other hand, the lowest EE was registered in the São Luiz Gonzaga-RS area (6.1), which can be justified by the low grain yield reached (Tables 1 and 8). The other areas evaluated in the southern region of Brazil obtained EE values very close, between 7 and 8 (Table 8). The highlight for this region is that when compared to other regions, seeds assume, on average, the main expense, followed by fuel and herbicides (Table 9).

On average general, fertilizers (29\%), seeds (23\%), herbicides (19\%), and fuels (15\%) accounted for the highest energy inputs in the assessed areas of soybean cultivation (Table 9). Similarly, other studies in the literature demonstrate that fertilizers (Mourad \& Walter, 2011; Riquetti, 2014), seeds (A. T. Campos et al., 2009; Riquetti, 2014), herbicides (Melo et al., 2007; A. T. Campos et al., 2009; Assenheimer et al., 2009; Riquetti, 2014), and fuels (A. T. Campos et al., 2009; Ferreira et al., 2014) accounted for the highest energy inputs in soybean cultivation in Brazil. 
Table 9

Main energy expenditure (\%) in the agricultural stage of soybean areas evaluated

\begin{tabular}{|c|c|c|c|c|c|c|c|c|c|c|}
\hline \multirow{3}{*}{ Area } & \multicolumn{10}{|c|}{---------- Main energy expenditure (\%) -------- } \\
\hline & $\mathrm{LH}^{*}$ & TMI & $\mathrm{F}$ & $\mathrm{Se}$ & Lim & SF & $\mathrm{HE}$ & PPP & PPD & TL \\
\hline & \multicolumn{10}{|c|}{----------------- Midwest region ------------------ } \\
\hline 1 & 0 & 1 & 11 & 17 & 1 & 33 & 27 & 5 & 1 & 2 \\
\hline 2 & 0 & 2 & 13 & 19 & 1 & 37 & 18 & 5 & 1 & 2 \\
\hline 3 & 0 & 2 & 13 & 21 & 1 & 24 & 25 & 10 & 2 & 2 \\
\hline 4 & 0 & 2 & 14 & 23 & 1 & 25 & 19 & 10 & 2 & 2 \\
\hline 5 & 0 & 2 & 11 & 16 & 1 & 24 & 38 & 3 & 1 & 2 \\
\hline 6 & 0 & 2 & 16 & 23 & 1 & 35 & 14 & 4 & 2 & 2 \\
\hline 7 & 1 & 4 & 24 & 24 & 1 & 28 & 13 & 0 & 2 & 2 \\
\hline 8 & 0 & 1 & 11 & 28 & 1 & 29 & 19 & 6 & 2 & 2 \\
\hline 9 & 0 & 3 & 18 & 24 & 1 & 30 & 19 & 1 & 1 & 2 \\
\hline 10 & 0 & 6 & 8 & 29 & 2 & 24 & 19 & 8 & 1 & 2 \\
\hline 11 & 0 & 6 & 7 & 23 & 1 & 32 & 18 & 8 & 2 & 2 \\
\hline 12 & 0 & 6 & 7 & 23 & 1 & 32 & 18 & 8 & 2 & 2 \\
\hline 13 & 1 & 10 & 14 & 20 & 2 & 25 & 18 & 7 & 1 & 2 \\
\hline 14 & 0 & 2 & 5 & 28 & 1 & 33 & 20 & 5 & 3 & 2 \\
\hline 15 & 0 & 5 & 14 & 21 & 1 & 28 & 23 & 2 & 2 & 2 \\
\hline 16 & 0 & 2 & 7 & 17 & 1 & 50 & 12 & 8 & 1 & 2 \\
\hline 17 & 0 & 2 & 6 & 31 & 1 & 35 & 16 & 4 & 2 & 2 \\
\hline 18 & 0 & 1 & 7 & 33 & 1 & 31 & 16 & 5 & 3 & 2 \\
\hline 19 & 0 & 3 & 13 & 23 & 1 & 38 & 16 & 1 & 2 & 2 \\
\hline 20 & 0 & 1 & 11 & 24 & 1 & 35 & 20 & 5 & 1 & 2 \\
\hline Mean: & 0.3 & 3.1 & 11.7 & 23.5 & 0.9 & 31.3 & 19.5 & 5.3 & 1.8 & 2.2 \\
\hline \multicolumn{11}{|c|}{--------------------- Northeast Region ------------------ } \\
\hline 21 & 0 & 2 & 17 & 19 & 1 & 38 & 13 & 4 & 2 & 2 \\
\hline 22 & 0 & 5 & 25 & 13 & 2 & 37 & 13 & 1 & 2 & 2 \\
\hline Mean: & 0.3 & 3.6 & 21.0 & 15.9 & 1.3 & 37.3 & 13.1 & 2.6 & 1.9 & 2.2 \\
\hline \multicolumn{11}{|c|}{ 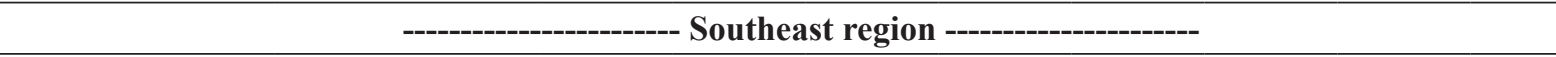 } \\
\hline 23 & 0 & 3 & 14 & 19 & 1 & 31 & 16 & 6 & 5 & 2 \\
\hline \multicolumn{11}{|c|}{ 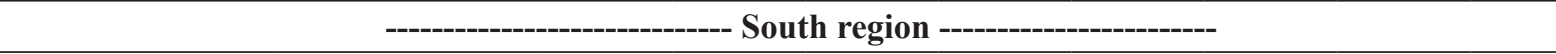 } \\
\hline 24 & 1 & 5 & 28 & 26 & 1 & 25 & 11 & 0 & 1 & 2 \\
\hline 25 & 1 & 3 & 21 & 25 & 1 & 23 & 20 & 3 & 1 & 2 \\
\hline 26 & 1 & 5 & 27 & 22 & 1 & 13 & 22 & 6 & 1 & 2 \\
\hline 27 & 1 & 5 & 28 & 22 & 1 & 14 & 20 & 6 & 1 & 2 \\
\hline 28 & 0 & 2 & 15 & 25 & 1 & 24 & 26 & 2 & 2 & 2 \\
\hline 29 & 0 & 2 & 17 & 26 & 1 & 16 & 30 & 5 & 1 & 2 \\
\hline Mean: & 0.5 & 3.8 & 22.5 & 24.2 & 0.8 & 19.1 & 21.5 & 3.6 & 1.2 & 2.2 \\
\hline Overall average: & 0.3 & 3 & 15 & 23 & 1 & 29 & 19 & 5 & 2 & 2 \\
\hline
\end{tabular}


In the present study, in general the main factor contributing to energy consumption in soybean production was the use of phosphate and potassium fertilizers (Table 9), which are usually applied at relatively higher amounts (Table 7) than those used in other crops and/or in countries with temperate climates. In this study, a mean of $87 \mathrm{~kg} \mathrm{ha}^{-1}$ year $^{-1}$ of $\mathrm{P}_{2} \mathrm{O}_{5}$ and $\mathrm{K}_{2} \mathrm{O}$ was applied (Table 7). The amounts of $\mathrm{P}_{2} \mathrm{O}_{5}$ and $\mathrm{K}_{2} \mathrm{O}$ applied in China, Argentina, and the USA are 41 and 6, 11 and 0 , and 20 and $31 \mathrm{~kg} \mathrm{ha}^{-1}$ year $^{-1}$, respectively (Food and Agricultural Policy Research Institute [FAPRI], 2012). There is a higher energy input associated with fertilizers in Brazil because of the limited availability of nutrients in the soil-plant system under Brazilian edaphoclimatic conditions, which requires higher additions of these fertilizers to compensate for this limitation (Mourad \& Walter, 2011). This low nutrient availability is due to the nature of tropical soils (principally Ferralsols), which are known to exhibit high adsorption of $\mathrm{P}$ as a result of the presence of oxidehydroxides of Fe and Al (M. Campos, Antonangelo, \& Alleoni, 2016), and naturally low K content, a consequence of their high degree of weathering (Camenzind, Hättenschwiler, Treseder, Lehmann, \& Rillig, 2018).

Considering this impact of fertilizers on the total energy used in the production of soybean, one way to reduce energy expenditure during production is to increase the efficiency of crop nutrient use. The increased efficiency of $\mathrm{P}$ and $\mathrm{K}$ fertilizer used (Teixeira, Silva, Sousa, Mattiello, \& Soares, 2016), and of green fertilizers that promote nutrient cycling (Silva, Oliveira, Merloti, \& Sá, 2017), and rooting promotion via co-inoculation with Bradyrhizobium and Azospirillum (Ferri, Braccini, Anghinoni, \& Pereira, 2017) are examples of techniques that have been indicated to increase the efficiency of $\mathrm{P}$ and $\mathrm{K}$ use by soybean and thereby to increase EE. However, in terms of energy, one should be careful if opting to replace synthetic fertilizers by other sources of these elements. The replacement of a source of synthetic fertilizer by a "natural" source with a similar content of the element considerably increases the costs, with additional machinery for transportation to the field, as a result of the low concentration of the nutrient in the alternative source (Hülsbergen et al., 2001; Riquetti, 2014). Further studies should assess the extent to which this replacement affects the final EE in soybean crops.

The seeds used for planting have a great impact on energy consumption because their production requires energy. In the present study, a value of 31.75 MJ per kg of seeds was used (Table 2). Therefore, in the entire soybean crop area, seeds were the second greatest contributor to energy use (Table 9). The high amount of energy embodied in seeds results from the inputs in their production is greater than the use of inputs in the production of grains for food (Mourad \& Walter, 2011; Riquetti, 2014). For example, the frequent application of fungicides and insecticides to avoid the propagation of pests and diseases by seeds is a common practice. Moreover, the energy used in the processing, classification, packaging, and storage of seeds is higher than that used in the production of grains for food (Mourad \& Walter, 2011; Riquetti, 2014). Therefore, technologies that improve the stages of seed production and/or reduce the use of energy during their agro-industrial development may contribute to increases in the $\mathrm{EE}$ of soybean crops.

The third greatest energy input to the soybean crop was associated with herbicides (Table 9). This is probably because of the high amount of energy used in their production, which varies between 262.8 and 418.3 $\mathrm{MJ} \mathrm{kg}^{1}$, depending on the formulation (Table $3)$. Thus, small amounts of the active ingredient in herbicides, even at moderate doses and application frequencies, lead to significant energy inputs into the production system (Table 6 and Table 9). There are three major strategies to increase the EE of soybean crops, in terms of reducing energy expenditures on herbicides: 1) adequate selection of herbicides and/or herbicide application, aiming 
for a more efficient herbicide use and reduction in applied concentrations; 2) improvement of herbicide production efficiency in the industry, aimed at reducing the amount of energy embodied in this input; 3 ) prioritization of crop management techniques and/or adoption of conservation systems, such as the direct seeding system, which reduce weed infestations and thus the use of herbicides.

Lastly, fuel use accounted for the fourth greatest energy expenditure in the assessed soybean areas (Table 9). Regardless of the assessed area, the operations that required the most fuel were plowing and/or harrowing, sowing, and harvesting (Table 4 and 5). Alternative strategies to reduce fuel energy inputs would be the improvement of its use through more efficient engines and multifunctional agricultural machinery (Riquetti, 2014).

\section{Conclusions}

For every MJ of energy consumed in the production of soybean crop, 6.1; 6.7; 7.1 and 7.2 MJ of energy were produced in the form of grain, respectively in the areas assessed in the Midwest, northeast, southeast and south regions of Brazil. On a general average, the main energy expenditure on soybean cultivation in different regions of Brazil was with fertilizers, seeds and herbicides.

The adverse weather conditions of the year / harvest evaluated in the south-central region of Brazil resulted in low soybean yields and consequently interfered with lower energy efficiency in these regions.

The evaluation of energy efficiency in soybean crops to be very representative must be carried out in different regions and edaphoclimatic conditions.

\section{References}

Alluvione, F., Moretti, B., Sacco D., \& Grignani, C. (2011). EUE (energy use efficiency) of cropping systems for a sustainable agriculture. Energy, 36(7), 4468-4481. doi: 10.1016/j.energy.2011.03.075
Assenheimer, A., Campos, A. T., \& Gonçalves, A. C., Jr. (2009). Analysis of energy use in soybean production under conventional and organic farming systems. Ambiência, 5(3), 443-455.

Beber, J. A. C. (1989). Energy efficiency and agricultural production processes in smallholdings. Dissertação de mestrado, Universidade Federal de Santa Maria, Santa Maria, RS, Brasil.

Boddey, R. M., Soares, L. H. B., Alves, B. J. R., \& Urquiaga, S. (2008). Bio-ethanol production in Brazil. In D. Pimentel, Biofuels, solar and wind as renewable energy systems (pp. 321-356). Dordrecht, Holanda: Springer.

Camenzind, T., Hättenschwiler, S., Treseder, K., Lehmann, A., \& Rillig, M. C. (2018). Nutrient limitation of soil microbial processes in tropical forests. Ecological Monographs, 88(1), 4-21. doi: 10.1002/ecm.1279

Campos, A. T., Klosowsk, E. S., Souza, C. V., Zanini, A., \& Prestes, T. M. V. (2009). Analysis of energy use in soybean production under no-tillage system. Global Science and Technology, 2(2), 38-44.

Campos, M., Antonangelo, J. A., \& Alleoni, L. R. F. (2016). Phosphorus sorption index in humid tropical soils. Soil \& Tillage Research, 156(1), 110-118. doi: 10.1016/j.still.2015.09.020

Capellesso, A. J., \& Cazella, A. A. (2013). Sustainability indicator of agroecosystems: a case study in areas of maize cultivation. Ciência Rural, 43(12), 22972303. doi: $10.1590 / \mathrm{S} 0103-84782013005000130$

Chen, R., Qin, Z., Han, J., Wang, M., Taheripour, F., \& Tyner, W. (2018). Life cycle energy and greenhouse gas emission effects of biodiesel in the United States with induced land use change impacts. Bioresource Technology, 251(1), 249-58. doi: 10.1016/j. biortech.2017.12.031

Comitre, V. (1993). Avaliação energética e aspectos econômicos da soja na região de Ribeirão Preto, SP. Dissertação de mestrado, Universidade Estadual de Campinas, Campinas, SP, Brasil.

Companhia Nacional de Abastecimento (2010). Custos da produção agrícola: a metodologia da CONAB. Recuperado de http://www.conab.gov.br/conabweb/ download/safra/custos.pdf

Companhia Nacional de Abastecimento (2014). Perspectiva para a Agropecuária, safra 2014/2015. Recuperado de http://www.conab.gov.br/ OlalaCMS/uploads/arquivos/14_09_10_18_03_00_ perspectivas_2014-15.pdf 
Cunha, J. P. B., Campos, A. T., Martins, F. G. L., Paula, V. R., Volpato, C. E. S., \& Silva, F. C. (2015). Demanda energética de diferentes manejos de solo no cultivo de milho. Bioscience Journal, 31(3), 808-817. doi: 10.14393/BJv31n3a2015-22431

Energy Research Office (2015). Balanço energético brasileiro: ano base 2014. Recuperado de http://www.mme.gov.br/ documents/10584/1143895/2.1+-+BEN+2015++Documento+Completo+em+ Portugu $\% \mathrm{C} 3 \% \mathrm{AAs}+$ +Ingl\%C3\%AAs+\%28PDF\%29/22602d8c-a366$4 \mathrm{~d} 16-\mathrm{a} 15 \mathrm{f}-\mathrm{f} 29933 \mathrm{e} 816 \mathrm{ff}$ ? version $=1.0$

Ferreira, F. F., Neumann, O. S., \& Hoffmann, R. (2014). Matrix analysis of energy and economic crop of rice, soy and wheat in intensive production system in Rio Grande do Sul, Brazil. Ciência Rural, 44(2), 380385. doi: 10.1590/S0103-84782013005000157

Ferri, G. C., Braccini, A. L., Anghinoni, F. B. G., \& Pereira, L. C. (2017). Effects of associated coinoculation of Bradyrhizobium japonicum with Azospirillum brasilense on soybean yield and growth. African Journal of Agricultural Research, 12(1), 6-11. doi: 10.5897/AJAR2016.11711

Food and Agricultural Policy Research Institute (2012). World agricultural outlook - application rates. Recuperado de http://www.fapri.iastate.edu/ outlook/2012/

Gomes, G. P. (2012). Balanço de energia em sistema de cultivo orgânico para produção de oleaginosas $e$ vegetais. Dissertação de mestrado, Universidade Estadual de Londrina, Londrina, PR, Brasil.

Hülsbergen, K. J., Feil, B., Biermann, S., Rathke, G. W., Kalk,W. D., \& Diepenbrock, W. A. (2001). Method of energy balancing in crop production and its application in a long-term fertilizer trial. Agriculture, Ecosystems \& Environment, 86(3), 303-321. doi: 10.1016/S0167-8809(00)00286-3

Macedônio, A. C., \& Picchioni, S. A. (1985). Métodos de cálculo do consumo de energia fóssil na produção agrícola. Curitiba, Brasil: Secretaria de Estado da Agricultura.

Melo, D., Pereira, J. O., Souza, E. G., Gabriel, A., Fo Nóbrega, L. H. P., \& Pinheiro, R., Neto. (2007). Energy balance of soybean and corn production systems in a farm of the west of Paraná, Brazil. Acta Scientiarum. Agronomy, 29(2), 173-178. doi: 10.4025/actasciagron.v29i2.233
Mourad, A. L., \& Walter, A. (2011). The energy balance of soybean biodiesel in Brazil: a case study. Biofuels, Bioproducts and Biorefining, 5(2), 185-197. doi: $10.1002 /$ bbb. 278

Pimentel, D. (1980). Handbook of energy utilization in agriculture. Boca Raton, US: CRC Press.

Quadros,K.R.,\&Kokuszka, R.(2007). Balanço energético em sistemas convencionais e agroecológicos de cultivo de feijão na região de Rebouças, no Paraná. Revista Brasileira de Agroecologia, 2(1), 50-54.

Riquetti, N. B. (2014). Produtividade, eficiência energética e econômica em semeadura cruzada de soja. Tese de doutorado, Universidade Estadual Paulista "Júlio de Mesquita Filho", Botucatu, SP, Brasil.

Sá, J. M., Urquiaga, S., Jantalia, C. P., Soares, L. H. B., Alves, B. J. R., \& Boddey, R. M. (2013). Energy balance for the production of grain, meat, and biofuel in specialized and mixed agrosystems. Pesquisa Agropecuária Brasileira, 48(10), 1323-1331. doi: 10.1590/S0100-204X2013001000003

Santos, H. P., \& Fontaneli, R. S. (2007). Energy conversion and balance in agricultural systems of maize production under no-tillage. In H. P. Santos, R. S. Fontaneli, S. T. Spera, Agricultural systems of maize production under no-tillage (pp. 297-312). Passo Fundo, RS, Brasil: EMBRAPA Trigo.

Santos, H. P., Fontaneli, R. S., Spera, S. T., \& Dreon, G. (2013). Energy conversion and balance of integrated crop livestock production systems under no tillage. Revista Brasileira Ciências Agrarias, 8(1), 1-7. doi: 10.5039/agraria.v8i1a1392

Santos, H. P., Spera, S. T., Fontaneli, R. S., \& Dreon, G. (2011). Evaluation of the cultural practices on energy conversion and balance. Revista Brasileira Ciências Agrarias, 6(4), 634-641. doi: 10.1590/S00 06-87052007000200014

Silva, M. S., Oliveira, G. R. F., Merloti, L. F., \& Sá, M. E. (2017). Accumulation of nutrients and dry matter produced by Crotalaria juncea cultived in Cerrado. Brazilian Journal of Chemical Engineering, 11(1), 26-36. doi: 10.18011/bioeng2017v11n1p26-36

Souza, J. L., Casali, V. W. D., Santos, R. H. S., \& Cecon, P. R. (2008). Energetic balance and sustainability analysis in the organic production of vegetable crops. Horticultura Brasileira, 26(4), 433-440. doi: 10. $1590 /$ S0102-05362008000400003 
Teixeira, R. S., Silva, I. R., Sousa, R. N., Mattiello, E. M., \& Soares, E. M. B. (2016). Organic acid coated-slow-release phosphorus fertilizers improve $\mathrm{P}$ availability and maize growth in a tropical soil. Journal of Plant Nutrition and Soil Science, 16(4), 1097-112. doi: 10.4067/S0718-95162016005000081

United States Department of Agriculture (2018). World agricultural supply and demand estimates. Virgínia, US: USDA.
Vieira, G. (2007). Avaliação energética e custo de produção da cana-de-açúcar (saccharum) do preparo de solo ao $5^{\circ}$ corte. Tese de doutorado, Universidade Estadual Paulista "Júlio de Mesquita Filho", Botucatu, SP, Brasil. 